\title{
La clase invertida "FLIPPED CLASSROOM" como recurso metodológico aplicado a la docencia de farmacología
}

\author{
Castillo García Ea , Ibáñez Torres La , León Bello Ga ${ }^{\mathrm{a}}$, Sanahuja Santafé MAª, Villagrasa \\ Sebastián $V^{\text {a }}$, Martínez-Solís I ${ }^{a}$
}

apepartamento de Farmacia. Universidad CEU Cardenal Herrera. C/ Ramón y Cajal. Valencia. ecastillo@uchceu.es

\begin{abstract}
Flipped classroom is a pedagogical model created in 2012 by Jonathan Bergmann and Aaron Sams, Chemistry teachers at Woodland Park High School (Woodland Park Colorado).

In this model, rather than taking up limited class time to explain a new concept (often via lecture), the teacher add a variety of learning material (videos, articles, podcast. etc.) to Blackboard before class. The objective is to introduce students to the concepts that they are going to study during class, freeing up class time for more collaborative activities. Learning material includes main concepts and ideas of the unit of study and it can be produced originally by the teacher or it can be acquired from internet. The activity has been carried out during the first semester of the academic year 2018-19 in the subjects Clinical Pharmacology and Pharmacology in Pharmacy and Medicine degrees, respectively. Acquired knowledge of students was evaluated by Kahoot tests and a survey was conducted to analyse the student's opinion about the activity. Kahoot results showed that 80 of the students pass the test. On the other hand, based on the results of the survey, the activity proved to be very interesting for the students. The flipped classroom helped them to clarify concepts and many of them considered that having previous theoretical background is important to understand the activity, although it is not essential.
\end{abstract}

Keywords: Flipped classroom, pedagogical model, instructional strategy.

\footnotetext{
Resumen

La clase invertida es un modelo pedagógico creado en 2012 por Jonathan Bergmann y Aaron Sams, profesores de Química en Woodland Park High School (Woodland Park Colorado).

En este modelo, el profesor, en lugar de utilizar el tiempo de la clase para introducir nueva materia (vía lección magistral), previamente a que tenga lugar la clase proporciona a los alumnos, a través de la plataforma virtual, material docente (videos, artículos, podcast, etc.) para ayudarles a clarificar la materia objeto de estudio, de manera que puede utilizar el tiempo de la clase para realizar actividades docentes colaborativas. El material docente debe
} 
La clase invertida "flipped classroom" como recurso metodológico aplicado a la docencia de farmacología

incluir las ideas principales de la unidad de estudio y puede ser creado por el profesor o descargado de internet.

Esta actividad se ha desarrollado en el primer cuatrimestre del curso 2018-19 en las asignaturas Farmacología Clínica en el Grado de Farmacia y Farmacología del Grado en Medicina. Basándonos en los resultados obtenidos, la actividad ha resultado muy interesante para los estudiantes. La clase invertida les ha ayudado a clarificar conceptos y algunos estudiantes han considerado que tener conocimientos previos es importante para realizar la actividad, aunque no es esencial.

Palabras clave: clase invertida, modelo pedagógico, estrategia instruccional.

\section{Introducción}

En un modelo de enseñanza tradicional, las competencias generales y especificas de las asignaturas cursadas se adquieren mediante clases presenciales, en las que el alumno dedica la mayor parte de su tiempo a escuchar al profesor; y mediante la realización de trabajos fuerea del horario de clases, los cuales pueden prestar dificultades en su resolución (Escudero 2009, Trillo 2018).

Aunque este modelo ha sido adoptado por la mayoría de docentes a lo largo de los años, ya en la década de los setenta se puso en duda su utilidad y eficacia. El pedagogo estadounidense Benjamin Bloom, consideró que la parte creativa no debía relegarse al trabajo en casa ya que ese tipo de aprendizaje suele ser el más complicado y requiere de un apoyo por parte del profesor. Trabajos más acuales como el de Enric Mazur, decano de Física Aplicada de la Universidad de Harvard, también rechazan la comunicación pasiva entre el profesor y el estudiante considerando necesario instaurar un aprendizaje entre pares, o peer instruction, que fomente un método más interactivo en el aula (Mazur E 2001).

En este sentido, en 2012, Jonathan Bergmann y Aaron Sams (profesores de química en Woodland Park High School, Woodland Park Colorado) desarrollaron una estrategia didáctica denominada Clase Invertida (Flipped classroom). Esta metodología se basa en facilitar a los alumnos los contenidos del temario (en forma de vídeos, libros o artículos) antes de la clase presencial a través del aulta virtual de la Universidad. De esta manera, los alumnos trabajan en casa los contenidos y aprovechan las horas de clase para resolver dudas en un ambiente más dinámico. Por otro lado, esta metodología permite al profesor saber en todo momento quién ha accedido y revisado la información en el aula virtual, así como centrar más la atención en las necesidades individuales de aprendizaje de cada estudiante (Bergmann 2012).

La 'clase invertida' presenta distintas ventajas para el alumno:

1. El uso de esta metodología permite que el alumno refuerce sus conocimientos ya que tiene un primer contacto con la unidad didáctica en casa y la repasa en clase mediante actividades dinámicas y con ayuda del profesor (Benitez 2017). Este método, permite que se invierta mayor tiempo en las categorías superiores de la taxonomía de Bloom en las clases.

(c)) BY-NC-ND 2019, Universitat Politècnica de València 


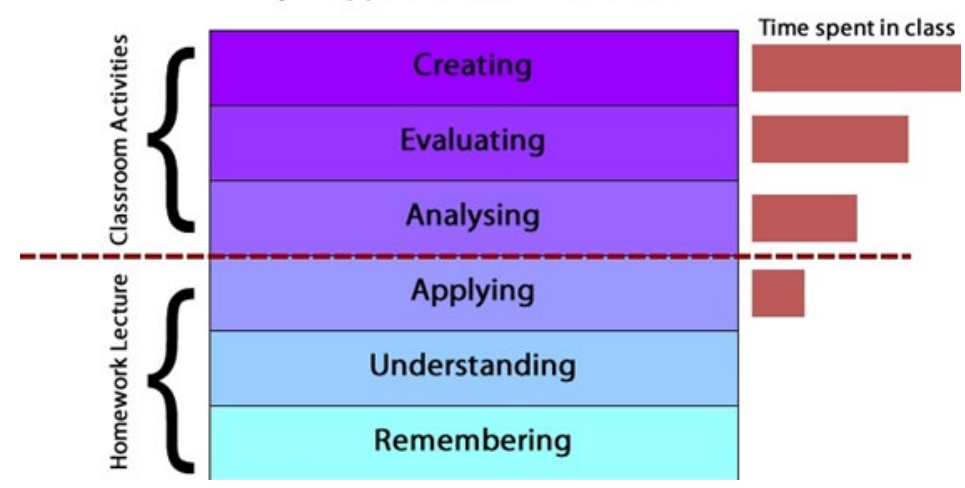

Fig. 1. Tiempo utilizado en la 'clase invertida' por taxonomía de Bloom (Tomado de Tourón J 2014))

2. La 'clase invertida' permite que el alumno aprenda según sus necesidades gracias a que puede utilizar "a demanda" el material subido al aula virtual (una pausa en el video-tutorial para tomar notas, o retroceder y aclarar cuando lo necesitan).

3. Por último, el empleo de esa metodología permite que el alumno adquiera otras competencias como la autonomía en el trabajo dentro y fuera del aula, la motivación hacia la asignatura, el compromiso con el grado, con el profesor y con sus compañeros, el desarrollo de habilidades de comunicación, empatía, asertividad y el trabajo coordinado con sus compañeros.

Así pues, el método de la clase invertida está acorde con la metodología defendida por El Espacio Europeo de Educación Superior (EEES) que apoya los métodos pedagógicos activos, participativos, colaborativos, en entornos de resolución de problemas y de desarrollo de proyectos, que se asimilan a un proceso continuo de 'entrenamiento'.

A pesar de la postura del EEES, en la práctica diaria de las aulas universitarias se sigue utilizando principalmente el método lección magistral-expositiva, utilizando los métodos activos 'actividades de evaluación continua' como seminarios o talleres prácticos. (Pérez Juste 2006). El modelo 'flipped classroom' puede presentarse como una herramienta que facilite el cambio de metodología pedagógica en las aulas universitarias, sustituyendo la lección magistral-expositiva por una enseñanza más didáctica que refuerza diversas competencias del alumno (Walsh 2013).

La asignatura Farmacología es una asignatura con gran cantidad de contenido teórico (nombres de fármacos, mecanismos de acción, efectos adversos, etc.) lo que la convierte en una materia a la cual los estudiantes deben dedicar mucho tiempo de estudio, y es, en sus grados correspondientes, una de las asignaturas con mayor número de alumnos suspensos. Por todo ello, pensamos en introducir esta metodología en la clase para al alumnado y facilitar el aprendizaje de los contenidos impartidos en la misma. 


\section{Objetivos}

El objetivo general de este proyecto es mejorar la calidad de los procesos de enseñanzaaprendizaje en la materia de Farmacología de las titulaciones de Farmacia y Medicina de la Universidad CEU Cardenal Herrera, adaptando el enfoque flipped classroom a uno de los bloques temáticos. Nos hemos planteado los siguientes objetivos específicos:

Conocer el grado de satisfacción del alumnado con respecto al modelo de clase invertida, y delimitar sus ventajas e inconvenientes desde el punto de vista tanto del alumnado como del profesor.

Potenciar el trabajo autónomo del alumno.

Mejorar las habilidades comunicativas de cada estudiante.

Favorecer el trabajo colaborativo y la labor de equipo.

\section{Desarrollo de la innovación}

La innovación se ha desarrollado en la asignaturas de Farmacología, concretamente Farmacología Clínica de tercer curso del Grado en Farmacia, y Pharmacology de tercer curso del Grado en Medicine, ambas titulaciones pertenecen a la Facultad de Ciencias de la Salud de la Universidad CEU Cardenal Herrera en Alfara del Patriarca, Valencia.

La asignatura de la titulación de Farmacia cuenta con 63 alumnos matriculados y la de Medicine con 75 alumnos.

Los profesores seleccionaron los temas de antibioticoterapia como el material de estudio para invertir las clases. En Farmacia se trató el tema de antibióticos del grupo quinolonas y en Medicine se realizaron tres sesiones con este tipo de actividad en las cuales se estudiaron antibióticos $\beta$-lactámicos, aminoglucósidos y glucopéptidos en la primera sesión, antibióticos macrólidos y tetraciclinas en la segunda y antibióticos del grupo quinolonas, sulfonamidas y trimetoprima en la tercera sesión.

Una semana antes de la clase presencial, los profesores incluyeron en la plataforma virtual de la Universidad (BLACKBOARD), dentro de cada una de estas asignaturas, vídeos (en los que se explicaba de manera gráfica los distintos mecanismos de acción de los antibióticos en estudio), artículos y un capítulo de libro sobre antibióticos (en los que se explicaba, además del mecanismo de acción, la farmacocinética, las reacciones adversas y las indicaciones de los antibióticos) y se avisó a los alumnos que deberían visualizar el material en casa antes de la próxima clase presencial.

En el aula, los profesores distribuyeron a los alumnos en grupos de 5-6 estudiantes (figura 2), uno de ellos es nombrado portavoz del grupo. 


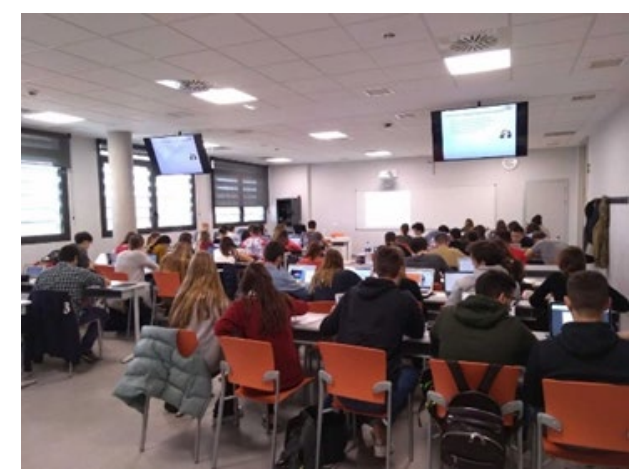

Fig.2. Alumnos distribuidos en grupos.

La actividad tiene una duración aproximada de 125 minutos, los cuales se dividieron en seis partes. En la figura 3 se muestra la estructura de la actividad, indicando el tiempo dedicado a cada parte de la misma.

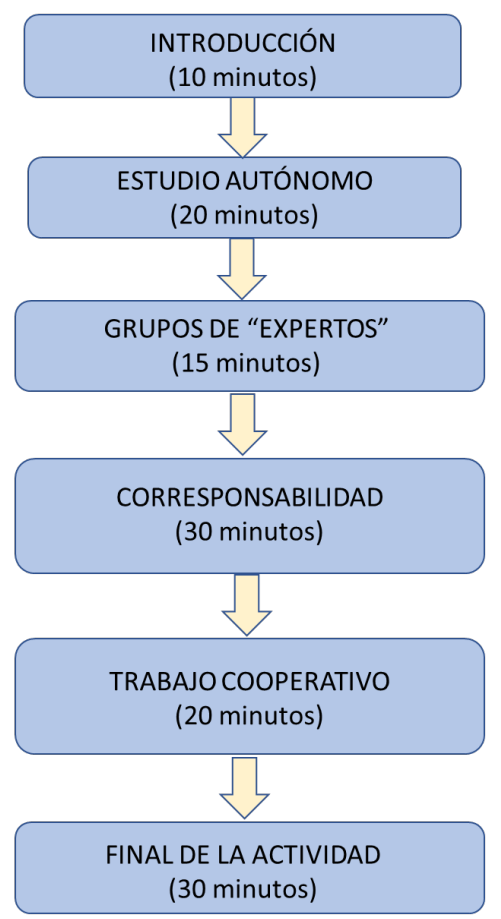

Fig.3. Estructura de la actividad

En la introducción el profesor introduce el tema y plantea 5 cuestiones, una para cada alumno del grupo. A continuación, cada estudiante busca la información de manera individual y resuelve su cuestión, durante este tiempo (20 minutos), los alumnos pueden preguntar al profesor las dudas que tengan. A continuación, se forman los grupos de expertos (todos los 
estudiantes con la misma cuestión se reúnen) para trabajar durante 15 minutos debatiendo y consensuando una respuesta definitiva, en esta parte de la actividad, el profesor pasa por los distintos grupos para asegurarse de que la información básica ha sido entendida. Esto, además, permite que los alumnos le consulten dudas en caso de que la información de cada alumno "experto" no coincida o sea contradictoria. En la fase de corresponsabilidad, cada estudiante vuelve a su grupo inicial y explica su cuestión a sus compañeros, de esta manera, todos los alumnos de un grupo conocerán la respuesta correcta correspondiente a cada una a las 5 preguntas iniciales.

Para finalizar, en la fase de trabajo cooperativo, entre todos los miembros del grupo elaboran el material en el que responden a todas las preguntas.

El final de la actividad consiste en realizar:

a) Un debate conjunto. A través de los portavoces, cada grupo expone los resultados de cada pregunta y se realizan comentarios finales de las cuestiones. Los grupos entregan las respuestas al profesor.

c) Un KAHOOT, con diez preguntas sobre la materia trabajada (se les entrega a los alumnos la puntuación obtenida en el mismo), que nos servirá para ver si los alumnos han alcanzado el grado de conocimiento esperado.

d) Una evaluación individual de la actividad mediante la encuesta que se muestra en la figura 3. La encuesta nos sirve para analizar la percepción que ha tenido el alumno respecto a su aprendizaje en esta sesión, valorando, entre otros aspectos, si considera que el tiempo, el material proporcionado, y su grado de aprendizaje eran adecuados. Los resultados de la misma serán utilizados para mejorar aquellos aspectos que hayan sido valorados de forma negativa por los alumnos, de manera que en el próximo curso podamos ir perfeccionando la implementación de esta nueva metodología. 


\begin{tabular}{|c|c|c|c|c|c|}
\hline & \begin{tabular}{|l|} 
Muy \\
inadecuado
\end{tabular} & \begin{tabular}{|l|} 
Algo \\
inadecuado
\end{tabular} & $\begin{array}{l}\text { Ni adecuado ni } \\
\text { inadecuado }\end{array}$ & Algo adecuado & Muy adecuado \\
\hline \multicolumn{6}{|l|}{$\begin{array}{l}\text { 1.- El material didáctico proporcionado para preparar la } \\
\text { clase lo considero }\end{array}$} \\
\hline \multicolumn{6}{|l|}{ 2.- El contenido de la clase me ha parecido } \\
\hline \multicolumn{6}{|l|}{ 3.- La metodología empleada me ha parecido } \\
\hline \multicolumn{6}{|l|}{$\begin{array}{l}\text { 4.- El tiempo invertido en el desarrollo de las actividades } \\
\text { en clase ha sido }\end{array}$} \\
\hline \multicolumn{6}{|l|}{$\begin{array}{l}\text { 5.- La organización y estructura de la clase me ha } \\
\text { parecido }\end{array}$} \\
\hline \multicolumn{6}{|l|}{$\begin{array}{l}\text { 6.- Los objetivos propuestos en esta sesión me han } \\
\text { parecido }\end{array}$} \\
\hline \multicolumn{6}{|l|}{$\begin{array}{l}\text { 7.- La documentación y las lecturas proporcionadas antes } \\
\text { de la clase me han parecido }\end{array}$} \\
\hline \multicolumn{6}{|l|}{$\begin{array}{l}\text { 8.- Las enseñanzas recibidas en esta sesión serán muy } \\
\text { útiles en mi desarrollo profesional }\end{array}$} \\
\hline \multicolumn{6}{|l|}{$\begin{array}{l}\text { 9.- La relación entre el profesor y los compañeros se ha } \\
\text { desarrollado en un clima favorable al aprendizaje }\end{array}$} \\
\hline \multicolumn{6}{|l|}{$\begin{array}{l}\text { 10.-El trabajo fundamentado en un aprendizaje más } \\
\text { activo me parece }\end{array}$} \\
\hline \multicolumn{6}{|l|}{$\begin{array}{l}\text { 11.- Mi percepción sobre el grado de aprendizaje en esta } \\
\text { sesión me ha parecido }\end{array}$} \\
\hline $\begin{array}{l}\text { 12.- Mi grado de satisfacción con la experiencia } \\
\text { educativa planteada en clase es }\end{array}$ & & & & & \\
\hline
\end{tabular}

Figura 3.- Encuesta adaptada de Melo L 2017.

\section{Resultados y discusión}

Realizaron la actividad el $81.5 \%$ de los alumnos matriculados en estas asignaturas (54 alumnos en Farmacia (85.7\%) y 58 alumnos de Medicine (77.3\%)). La asistencia a la actividad no era obligatoria, por lo que el alto porcentaje de asistencia muestra el interés que despertó la misma entre el alumnado de estas asignaturas.

En las preguntas realizadas mediante Kahoot se obtuvo que un $84 \%$ de los alumnos de Medicine contestó bien el 50\% o más de las preguntas mientras que en Farmacia representó un $95 \%$. Es de destacar que en Farmacia, el 76\% de los alumnos contestó bien todas las preguntas lo que indica que los alumnos han adquirido los conocimientos previstos en esta sesión.

Los resultados de las encuestas de valoración de la actividad se muestran en la figura 4.
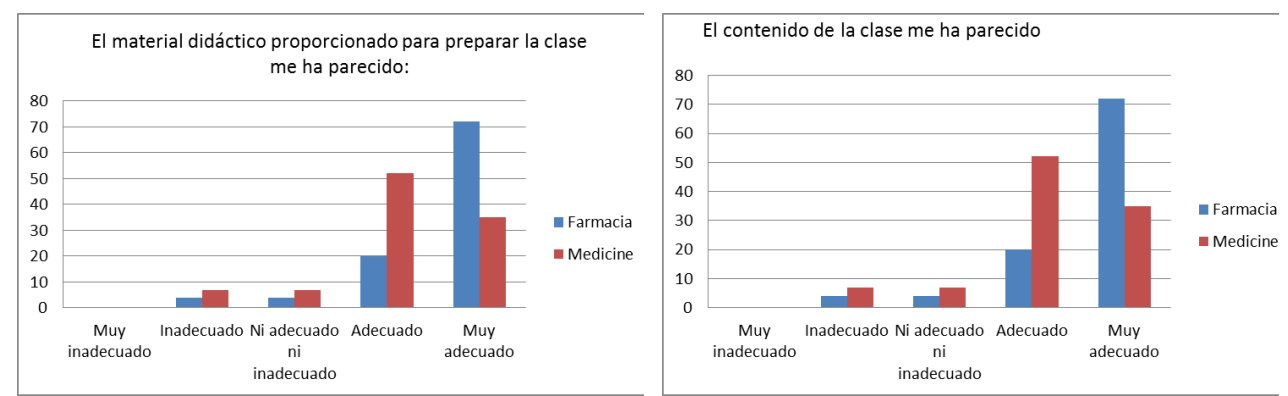
La clase invertida "flipped classroom" como recurso metodológico aplicado a la docencia de farmacología
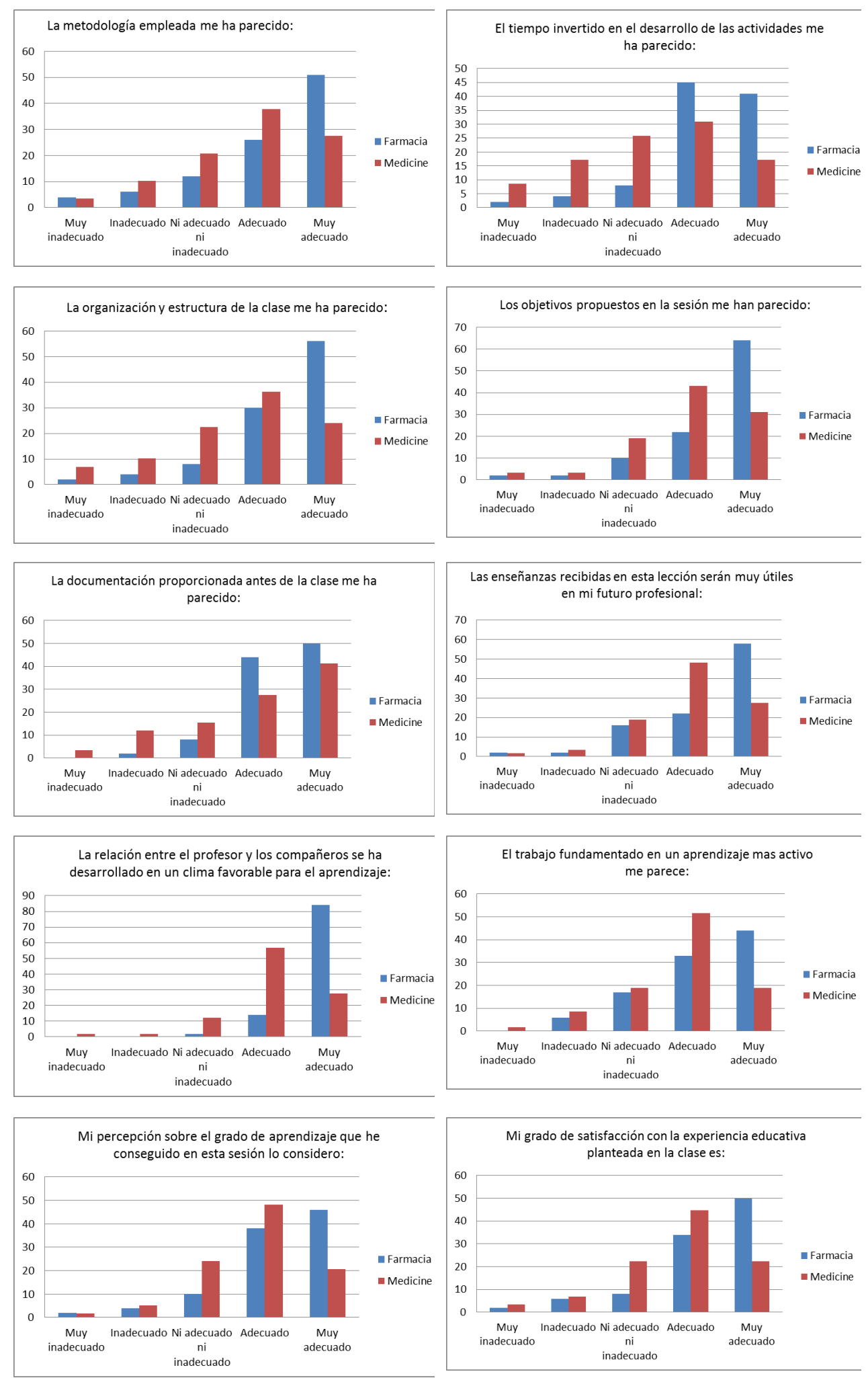

Figura 4. Resultados de las encuestas de satisfacción con la actividad.

(cc) EY-NC-ND 2019, Universitat Politècnica de València 
Como se observa en las graficas, la mayoría de alumnos han valorado como muy adecuadas o adecuadas todas las preguntas relacionadas con esta actividad.

Comparando los resultados de las encuestas entre las dos titulaciones, destaca que la mayoría de los alumnos de Farmacia valoran de forma muy adecuada todas las preguntas menos la del tiempo empleado para desarrollar la actividad que lo valoran de forma adecuada, mientras la que la mayoría de los alumnos de Medicine valorán de forma adecuada todas las preguntas menos la de la documentación entregada que la valoran de forma muy adecuada.

Además, llama la atención que un $76 \%$ de todos los alumnos que han realizado la actividad consideran que el trabajo basado en un aprendizaje más activo les parece adecuado o muy adecuado, mientras que un $17 \%$ no lo consideran ni adecuado ni inadecuado y sólo un $8 \%$ algo inadecuado.

Por último, $67.2 \%$ de los alumnos de Medicine y $84 \%$ de los alumnos de Farmacia consideran que su grado de satisfacción con la experiencia educativa planteada es adecuado o muy adecuado.

Estos resultados demuestran que esta nueva metodología es bien aceptada por los alumnos ya que todas las preguntas de la encuesta han sido valoradas como muy adecuadas o adecuadas. Además, los resutlados demuestran que el abandono de la clase magistral en favor del desarrollo de actividades más dinámicas y activas, como es la Clase Invertida, favorece el aprendizaje ya que más del $80 \%$ de los alumnos de ambas titulaciones consiguió contestar correctamente a más de la mitad de las preguntas planteadas durante las sesiones.

Claramente, si comparamos esta metodología aplicada en este estudio con la clase magistral tradicional podemos decir que se logran los objetivos planteados: se ha potenciado el trabajo autónomo del alumno, ya que ha tenido que buscar información para tratar de responder a las cuestiones planteadas, se han mejorado sus habilidades comunicativas, puesto que han tenido que exponer sus conclusiones y han sido coregidos por un profesor según haya sido su forma de exponer, se ha favorecido el trabajo colaborativo y la labor de equipo teniendo en cuenta como ha sido la metodología empleada.

\section{Conclusiones}

La actividad se ha realizado de forma satisfactoria en las dos titulaciones de Salud. Un 80\% de los alumnos han aprobado el test realizado mediante el Kahoot.

Un $61 \%$ del total de alumnos que han realizado la actividad consideran que su grado de satisfacción con la experiencia educativa planteada es muy adecuado, y un 39\% la consideran adecuada.

La actividad ha potenciado el trabajo autónomo del alumno (fase de estudio autónomo), las habilidades comunicativas con sus compañeros (fase de corresponsabilidad) y ha favorecerido el trabajo colaborativo y la labor de equipo (fase de trabajo cooperativo). 
La clase invertida "flipped classroom" como recurso metodológico aplicado a la docencia de farmacología

El tiempo asignado a la actividad (120 minutos) es insuficiente para tratar con profundidad cada uno de los apartados, para próximas sesiones ampliaremos el tiempo dedicado a esta actividad, puesto que un $30 \%$ de alumnos considera que no es adecuado.

Los resultados obtenidos demuestran que el empleo de la Clase Invertida incrementa los conocimientos del alumno relacionados con una materia determinada, lo que les permite superar de manera satisfactoria los test de evaluación. Además, los alumnos valoran de manera positiva el empleo de esta metodología para el estudio de la Farmacología.

\section{Referencias}

BENÍTEZ, M. (2017).’Flipped-classroom para las mentes del mañana en el aula de Geografía e Historia” en Martínez, R., García-Moris R, y García C.R. Investigación en didáctica de las ciencias sociales. Retos, preguntas y líneas de investigación. Cordoba: Universidad de Córdoba.

BERGMANN, J. y SAMS, A. (2012). Flip Your Classroom: Reach Every Student in Every Class Every Day. Virginia: ASCD.

BLACKBOARD. <https://www.blackboard.com/index.html>[Consultado el 13 de Mayo de 20019]

BLOOM, B., ENGELHART, M.D., HILL, W.H., FURST, E.J y KRATHWOHL, D.R. (1971). Taxonomía de los objetivos de la educación: la clasificación de las metas educacionales : manuales I y II. Buenos Aires: Centro Regional de Ayuda Técnica: Agencia para el Desarrollo Internacional.

ESCUDERO MUÑOZ, J.M. (2009) "Las competencias profesionales y la formación universitarias: posibilidades y riesgos" en Pedagogía social: revista interuniversitaria, vol.16, p. 1989-9742.

KAHOOT. < https://kahoot.com/>[Consultado el 13 de Mayo de 20019]

MAZUR, E. y CROUCH, C.H. (2001) "Peer Instruction: Ten years of experience and results" en American Journal of Physics, vol.69, p. 970-977.

MELO L. y SÁNCHEZ R. (2017) “Análisis de las percepciones de los alumnos sobre la metodología flipped classroom para la enseñanza de técnicas avanzadas en laboratorios de análisis de residuos de medicamentos veterinarios y contaminante" en Educación Química, vol. 28, p. 30-37.

PÉREZ JUSTE, R. (2006) Evaluación de programas educativos. Madrid: La Muralla. 
TOURON, J., SANTIAGO, R., y DIEZ, A. (2014) “The flipped classroom. Como convertir la escuela en un espacio de aprendizaje". Barcelona: Digital Text.

TRILLO ALONSO, J.F, ZABALZA BERAZA, M.A, y ZABALZA CERDEIRIÑA, M.A. (2018) “¿Hemos cambiado? La perspectiva de los profesores eméritos sobre el cambio educativo en la universidad?" en Educatio siglo XXI: Revista de la Facultad de Educación, vol. 36 , p. 255-276.

WALSH, K. (2013) "Flipped Classroom Panel Discussion Provides Rich Insights into a Powerful Teaching Technique" en Emergingedtech, 16 de Junio. $<$ http://www.emergingedtech.com/2013/06/flipped-classroom-panel-discussion-providesrich-insights-into-a-powerful-teaching-technique/> [Consulta: 13 de septiembre 2018] 